\title{
Domain wall motion in nanowires
}

\author{
R. WIESER*, U. NOWAK and K.D. USADEL \\ Institute of Physics, University of Duisburg Essen, \\ Duisburg Campus, 47048 Duisburg, Germany
}

\begin{abstract}
We investigated the motion of domain walls in ferromagnetic cylindrical nanowires by solving the Landau Lifshitz Gilbert equation numerically for a classical spin model in which energy contributions from exchange, crystalline anisotropy, dipole dipole interactions, and a driving magnetic field are considered. Depending on the diameter, either transverse domain walls or vortex walls are found. A transverse domain wall is observed for diameters smaller than the exchange length of the given system. In this case, the system effectively behaves one dimensionally and the domain wall velocity agrees with the result of Slonczewski for one dimensional walls. For larger diameters, a crossover to a vortex wall sets in which enhances the domain wall velocity drastically. For a vortex wall the domain wall velocity is described by the Walker formula.
\end{abstract}

Keywords: Domain wall motion; Classical spin model; LLG; Langevin dynamics

\section{Introduction}

Arrays of magnetic nanowires are possible candidates for patterned magnetic storage media [1, 2]. For these nanowires and also for other future magneto-electronic devices an understanding of domain wall motion and mobility is important for the controlled switching of the nanostructure. In a recent experiment the velocity of a domain wall in a $\mathrm{NiFe} / \mathrm{Cu} / \mathrm{NiFe}$ trilayer was investigated using the GMR effect [3]. The measured velocities were compared with the Landau-Lifshitz formula for domain wall motion [4]. This comparison was used to determine the damping constant of the trilayer, a quantity which is usually not known a priori. However, several formulae for the velocity of a domain wall can be found in the literature [4-8], which are derived in different limits but all of them in (quasi) one-dimensional models neglecting the possible influence of non-uniform spin structures within the domain wall. Thus the question arises as to what extent these formulae are applicable to real three-dimensional domain structures. To shed some light onto this problem we investigate numerically the domain wall mobility in nanowires starting from a three-dimensional local spin model.

*Corresponding author. E mail: robert@thp.uni duisburg de 


\section{Model and simulations}

In the following we consider a classical spin model with energy contributions from exchange, crystalline anisotropy, dipole-dipole interactions, and a driving magnetic field. Such a spin model for the description of magnetic nanostructures can be justified following different lines, see, e.g., Nowak [9]: on the one hand, it is the classical limit of a quantum mechanical localized spin model, and, on the other, it might be interpreted as the discretized version of a micromagnetic continuum model, where the charge distribution for a single cell of the discretized lattice is approximated by a point dipole. For certain magnetic systems their description in terms of a lattice of magnetic moments may even be based on the mesoscopic structure of the material, especially when a particulate medium is described.

However, our intention is not to describe a particular material, but to investigate a general model Hamiltonian:

$$
\begin{aligned}
\mathcal{H}= & -J \sum_{\langle i j\rangle} S_{i} \cdot S_{j}-\mu_{\mathrm{s}} B \cdot \sum_{i} S_{i}-D_{\mathrm{e}} \sum_{i}\left(S_{i}^{z}\right)^{2} \\
& -\omega \sum_{i<j} \frac{3\left(S_{i} \cdot e_{i j}\right)\left(e_{i j} \cdot S_{j}\right)-S_{i} \cdot S_{j}}{r_{i j}^{3}}
\end{aligned}
$$

where $S_{i}$ are three-dimensional magnetic moments of unit length on a cubic lattice.

The first sum is the ferromagnetic exchange between nearest neighbours with coupling constant $J$. The second sum is the coupling of the spins to an external magnetic field $B$, the third sum represents a uniaxial anisotropy, here with $D_{\mathrm{e}}>0$ favouring the $z$ axis as the easy axis of the system, and the last sum is the dipolar interaction, where $w=\mu_{0} \mu_{\mathrm{s}}^{2} /\left(4 \pi a^{3}\right)$ describes the strength of the dipole-dipole interaction. $e_{i j}$ are unit vectors pointing from lattice site $i$ to $j$ and $r_{i j}$ is the distance between these lattice sites in units of the lattice constant $a$.

The underlying equation of motion for the magnetic moments we consider in the following is the Landau-Lifshitz-Gilbert (LLG) equation,

$$
\frac{\partial S_{i}}{\partial t}=-\frac{\gamma}{\left(1+\alpha^{2}\right) \mu_{\mathrm{s}}} S_{i} \times\left[H_{i}(t)+\alpha S_{i} \times H_{i}(t)\right]
$$

with the gyromagnetic ratio $\gamma=1.76 \times 10^{11}(\mathrm{Ts})^{-1}$, the dimensionless damping constant $\alpha$ (after Gilbert), and the internal field $H_{i}(t)=-\partial \mathcal{H} / \partial S_{i}$.

In the following we present results from the simulation of cylindrical systems parallel to the $z$ axis with a length of 256 lattice sites and diameter $d=8$. Our systems are defined on a cubic lattice and consist of dipoles at those lattice sites which reside within the cylinder of given diameter and length. Due to shape as well as crystalline anisotropy the equilibrium magnetization is aligned with the long axis of the system. However, we start the simulation with an abrupt, head-to-head domain wall as the initial configuration, letting the wall relax until a stable state is reached. The distance of the initial wall position from the end is approximately one-third of the system length. Then we switch on the driving magnetic field $B$ along the easy axis and wait until a stationary state is reached for some time interval in which the velocity $v$ of the wall is constant while the wall is moving through the central part of the wire. We calculate the domain wall velocity from the magnetization versus time data, averaged over a period of time where no influence of the finite system size on the domain wall can be observed, i.e. until the wall approaches the other end of the wire. 


\section{Results and discussion}

Depending on the ratio $\omega / J$, either transverse domain walls or vortex domain walls are found. For the static limit $(B=0)$, representative spin configurations are shown in figure 1 . The important length scale is the so-called exchange length $d_{\mathrm{ex}} / a=\pi(J /[6 \omega \zeta(3)])^{1 / 2}$, where $\zeta(3) \approx 1.2$ is Riemann's Zeta function (for the exchange length in continuum theory, $3 \zeta(3)$ is replaced by $\pi$ ). The transverse domain wall is observed for wire diameters smaller than, and the vortex domain wall for wire diameters larger than, the exchange length [10-12].

The literature reports different equations for the velocity of domain walls in certain limits. One of them is the Walker equation [6],

$$
v=\frac{\gamma B a}{\alpha} \sqrt{\frac{J}{2\left(D_{\mathrm{e}}+D_{\mathrm{h}} \sin ^{2} \phi\right)}},
$$

which, in the limit $\phi=0$, was first calculated by Landau and Lifshitz [4]. The second equation was given by Malozemoff and Slonczewski [5],

$$
v=\frac{\gamma B a}{\alpha+1 / \alpha} \sqrt{\frac{J}{2 D_{\mathrm{e}}} .}
$$

The difference between these equations is in the dependence on the damping constant $\alpha$. Both equations are calculated by solving the Landau-Lifshitz-Gilbert equation in different limits; for a review, see Malozemoff and Slonczewski [5]. Figure 2 shows the two possible paths for the reversal of a spin. Path (1) is restricted to one plane as in the case of steady-state motion, while path (2) is dominated by precession.

The case of steady-state motion is described by the Walker equation (equation (3)), and the more general case with precession is described by the Slonczewski equation (equation (4)). Both equations were derived for one-dimensional systems, but they can also be used to calculate the velocity in three-dimensional nanowires [12].

For a transverse domain wall we found precessional motion. Figure 3 shows a moving transverse domain wall for three equidistant times. The precession of the
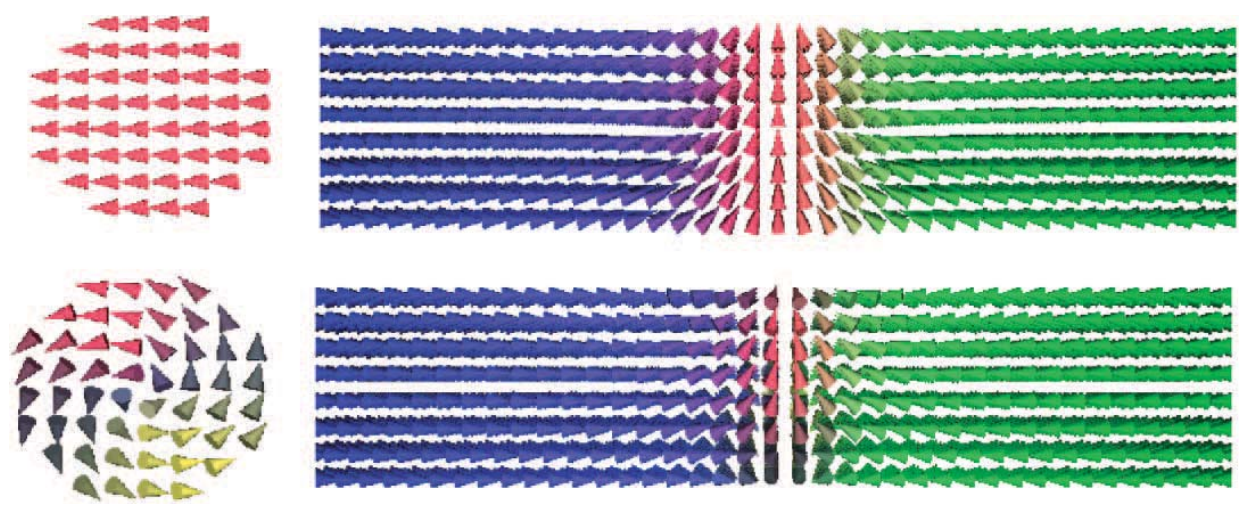

Figure 1. Snapshots of a transverse domain wall $\left(\begin{array}{lll}\omega / J & 0.0, D_{\mathrm{e}} / J & 0.05\end{array}\right)$ and a vortex domain wall $\left(\omega / J \quad 0.2, D_{\mathrm{e}} / J \quad 0.05\right)$. The wire diameter $d \quad 8$ is kept constant, while the exchange length is varied. 


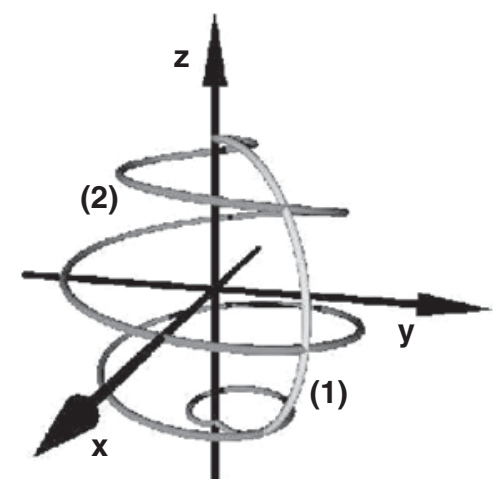

Figure 2. Different paths for the reversal of a spin: (1) direct reversal restricted to one plane, (2) precessional reversal.

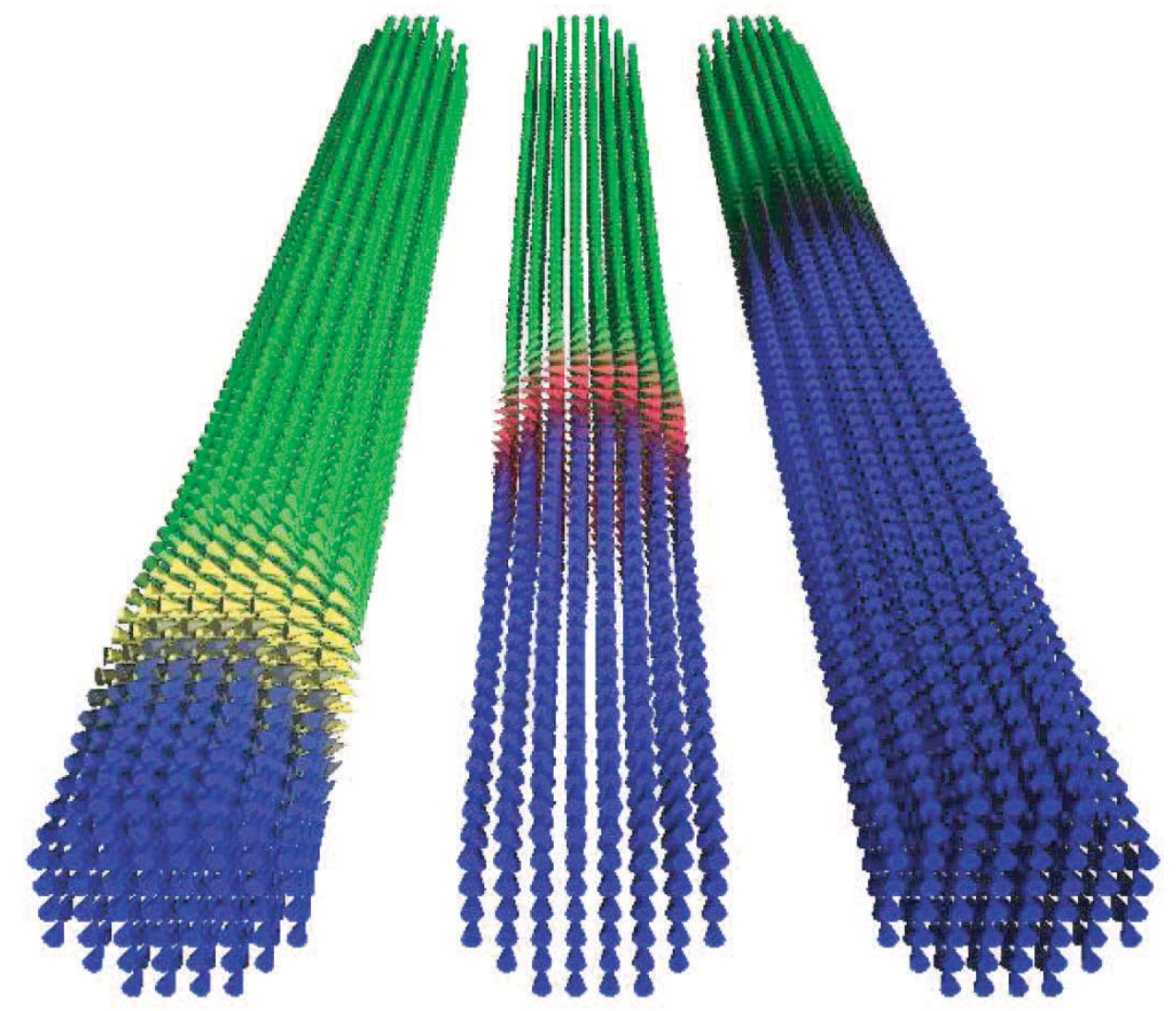

Figure 3. Snapshots of a transverse domain wall during motion $\left(\mu_{\mathrm{s}} B / J \quad 0.1, \alpha \quad 1\right)$ at three equidistant times. The precession of the spins during reversal leads to precession of the domain wall.

wall follows from the fact that the motion of each single spin consists of precession and relaxation with no restriction to a single plane (path (2) in figure 2). As shown numerically in this case, the domain wall velocity is well described by the Slonczewski equation (equation (4)) [12]. 


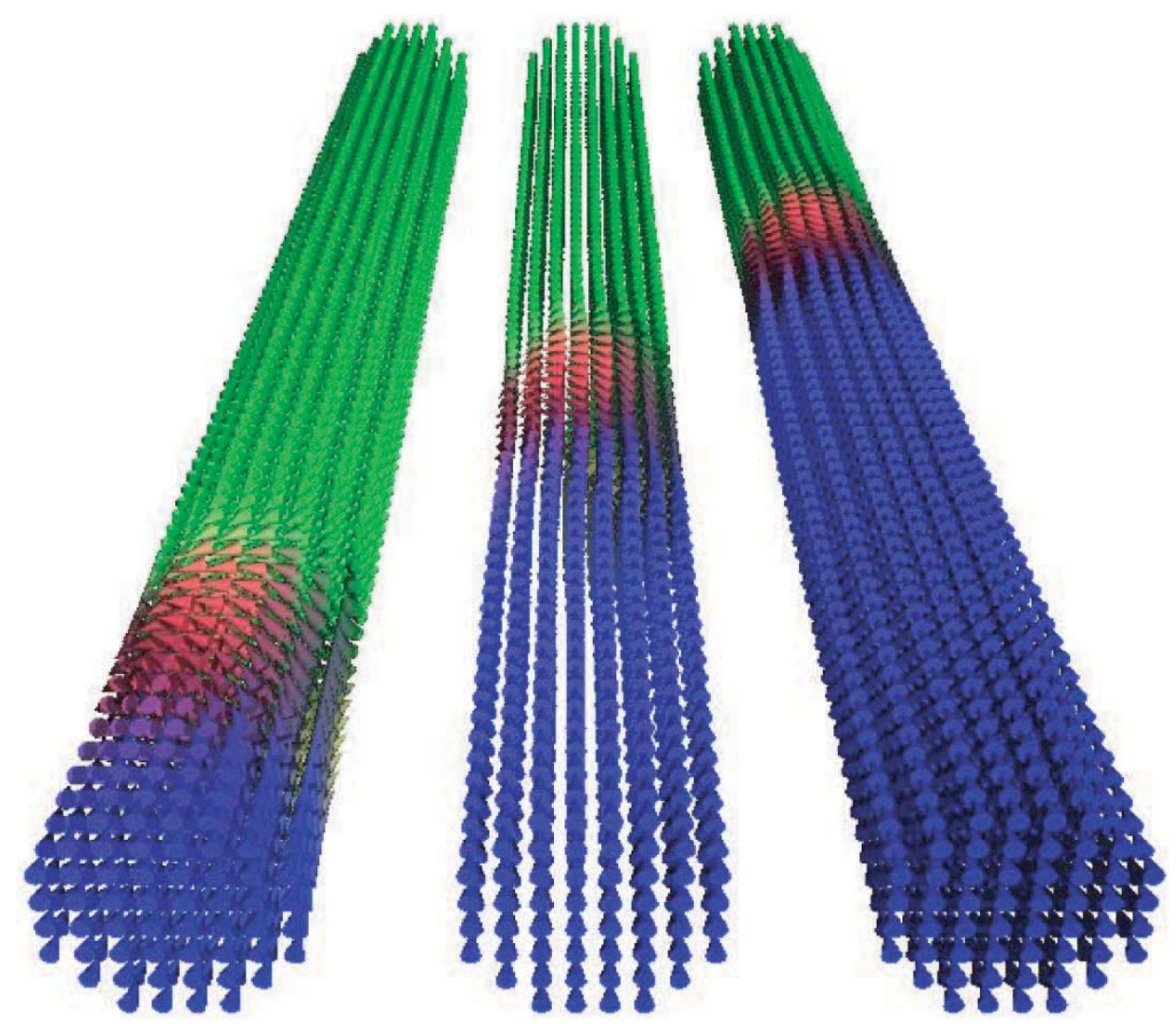

Figure 4. Snapshots of a vortex domain wall during motion $\left(\begin{array}{llll}\mu_{\mathrm{s}} B / J & 0.1, \alpha & 1\end{array}\right)$ at three equidistant times. The vortex inside the domain wall remains stable.

For a vortex domain wall the situation is different, and the velocity is well described by the Walker formula (equation (3)) [12]. Figure 4 shows snapshots of a moving vortex domain wall for three equidistant times. For a qualitative understanding of the spin motion in the case of a vortex domain wall, we note that the motion of the spins within each spin chain, parallel to the wire axis, is restricted to a certain plane passing through the spin positions. For spins at the surface of the cylinder, and in the limit of small driving fields, these planes are tangential to the surface of the cylinder. The force that keeps the spin motion of each spin chain in this plane is the energetic principle that forms the vortex, i.e. the combination of exchange and dipolar interactions.

\section{Summary}

In good agreement with prior work on cylindrical nanowires we have found different wall structures, transverse and vortex domain walls, depending on the diameter of the system compared with the exchange length of the given material.

In both cases the wall velocities determined numerically are in agreement with the simple analytical expressions derived in the one-dimensional limit. While for the transverse domain wall the velocity is described by the formula of Slonczewski, the 
vortex domain wall is described by the formula of Walker. The main difference between these formulae is the dependence on the damping constant $\alpha$. The reason for this difference is the reversal process during domain wall motion. In the case of the vortex domain wall the spin motion is completely within one single plane, while this is not the case for a transverse domain wall where precession leads to three-dimensional spin motion.

\section{Acknowledgment}

We acknowledge the support by the Deutsche Forschungsgemeinschaft (SFB 491).

\section{References}

[1] C.A. Ross, R.W. Chantrell, M. Hwang, M. Farhoud et al., Incoherent magnetization reversal in $30 \mathrm{~nm}$ Ni particles. Phys. Rev. B 6214252 (2000).

[2] K. Nielsch, R.B. Wehrspohn, J. Barthel, J. Kirschner et al., High density hexagonal nickel nanowire array. J. Magn. Magn. Mater. 249234 (2002).

[3] T. Ono, H. Miyajima, K. Shigeto, K. Mibu et al., Propagation of a magnetic domain wall in a submicron magnetic wire. Science 284468 (1999).

[4] D.L. Landau and E. Lifshitz, On the theory of the dispersion of magnetic permeability in ferromagnetic bodies. Phys. Z. Sowjetunion 8153 (1935)

[5] J.F. Dillon, In G.T. Rado and H. Suhl (Eds), Magnetism, Vol. 1. (Academic Press, New York, 1963), p. 149.

[6] N.L. Schryer and L.R. Walker The motion of $180^{\circ}$ domain walls in uniform de magnetic fields. J. Appl. Phys. 455406 (1974).

[7] A.P. Malozemoff and J.C. Slonczewski, Magnetic Domain Walls in Bubble Materials (Academic Press, New York, 1979).

[8] D.A. Garanin, Dynamics of elliptic domain walls. Physica A 178467 (1991).

[9] U. Nowak, Thermally activated reversal in magnetic nanostructures. In D. Stauffer (Ed.) Annual Reviews of Computational Physics IX (World Scientific, Singapore, 2001) pp. 105.

[10] H. Forster, T. Schrefl, D. Suess, W. Scholz et al., Domain wall motion in nano wires using moving grids. J. Appl. Phys. 916914 (2002).

[11] R. Hertel and J. Kirschner, Magnetization reversal dynamics in nickel nanowires. Physica B 343206 (2004).

[12] R. Wieser, U. Nowak and K.D. Usadel, Domain wall mobility in nanowires: transverse vs. vortex walls. Phys. Rev. B 69064401 (2004). 